\title{
Caracterização de propriedades tecnológicas de quatro folhosas deterioradas por térmitas do gênero Nasutitermes
}

\author{
Ezequiel GALLIO ${ }^{*}$, Sabrina Finatto MACHADO ${ }^{1}$, Francislene Junia Telles da SILVA ${ }^{1}$, \\ Nidria Dias CRUZ ${ }^{1}$, Darci Alberto GATTO ${ }^{2}$ \\ ${ }^{1}$ Centro de Desenvolvimento Tecnológico, Universidade Federal de Pelotas, Pelotas, RS, Brasil \\ ${ }^{2}$ Centro de Engenharias, Universidade Federal de Pelotas, Pelotas, RS, Brasil \\ *E-mail: egeng.florestal@gmail.com
}

Recebido em setembro/2017; Aceito em junho/2018

\begin{abstract}
RESUMO: O objetivo deste trabalho consistiu em analisar a deterioração causada por térmitas em madeiras de Mezilaurus itauba (Meisn.) Taub. ex Mez, Cordia americana (L.) Gottshling \& J. E. Mill., Melia azedarach L. e Parapiptadenia rigida (Benth.) Brenan, após ensaio de preferência alimentar. Foram confeccionados 20 corpos de prova de cada espécie, com dimensões de 15 x 15 × $260 \mathrm{~mm}$ (tangencial x radial x longitudinal). Os parâmetros tecnológicos avaliados foram a massa específica básica $(\rho b)$, a perda de massa $(\mathrm{PM})$, o módulo de elasticidade (MOE) e ruptura (MOR) provenientes do ensaio de flexão estática e a dureza. Finalizado o ensaio, a espécie com menor perda de massa foi a Melia azedarach, possivelmente devido à composição dos seus extrativos. Para as espécies Cordia americana e Parapiptadenia rigida, quanto às propriedades mecânicas, verificou-se redução significativa somente no MOE das amostras deterioradas em comparação com as do grupo controle. Conclui-se, portanto, que, a maior resistência da espécie Melia azedarach está associada à sua composição química, sendo que em quase todas as espécies madeireiras houve decréscimo nos parâmetros mecânicos (MOE, MOR e dureza Janka) devido ao ataque dos térmitas Nasutitermes.
\end{abstract}

Palavras-chave: biodeterioração, cupins, propriedades tecnológicas, espécies florestais.

\section{Technological properties characterization of four hardwoods deteriorated by gender Nasutitermes termites}

\begin{abstract}
The objective of this work was to analyze the deterioration caused by termites in woods of Mezilaurus itauba (Meisn.) Taub. ex Mez, Cordia americana (L.) Gottshling \& J. E. Mill., Melia azedarach L. and Parapiptadenia rigida (Benth.) Brenan, after food preference assay. Were made 20 test specimens of each species, with dimensions of $15 \times 15 \times 260 \mathrm{~mm}$ (tangential x radial x longitudinal). The physical parameters were the basic mass $(\rho b)$, the mass loss (PM), the modulus of elasticity (MOE) and rupture (MOR) from the static bending test and Janka hardness. After the test, the species with the lowest mass loss was Melia azedarach, possibly due to your extractives composition. For the species Cordia americana and Parapiptadenia rigida, about the mechanical properties, it was observed significant reduction only in the MOE of deteriorated samples compared to the control group. It's concluded that the Melia azedarach specie higher resistance it's associated with your chemical composition, and in almost all wood species there was a decrease in mechanical parameters (MOE, MOR and Janka hardness) due to attack of Nasutitermes termites.
\end{abstract}

Keywords: biodeterioration, termites, technological properties, forest species.

\section{INTRODUÇÃO}

As espécies Cordia americana (L.) Gottshling \& J. E. Mill., Melia azedarach L., Mezilaurus itauba (Meisn.) Taub. ex Mez. e Parapiptadenia rigida (Benth.) Brenan, podem ser empregadas em diversas finalidades, tais como: madeira serrada para construção civil externa e interna, construção rural, movelaria, medicina, ornamentação e energia (CARVALHO, 2002; CARVALHO, 2003; RODRIGUES et al., 2007; VENSON et al., 2008). As espécies madeireiras apresentam características únicas, e para otimizar seu uso, são necessários estudos visando à determinação de suas propriedades tecnológicas.

Outrossim, a durabilidade natural é uma variável relevante a ser considerada na seleção de uma espécie florestal. Essa propriedade é influenciada por outras variáveis, tais como: o ambiente de utilização do material, a resistência natural da madeira e tipos de agentes xilófagos aos quais os produtos madeireiros ficarão expostos (ARAUJO et al., 2012).

Esses organismos, reconhecem nos constituintes da madeira uma fonte de alimentos, destacando-se dentre eles os insetos da ordem Isoptera (CORASSA et al., 2014), os cupins ou térmitas, com destaque para os cupins do gênero Nasutitermes em razão da ampla distribuição no território nacional e ataque a diferentes classes de madeiras (STALLBAUN et al., 2017).

As térmitas possuem elevada capacidade de desenvolvimento em ambientes aos quais as condições lhes sejam favoráveis (MOTTA et al., 2013), gerando prejuízos socioeconômicos relevantes. A deterioração das peças de madeira compromete sua estrutura física, acarretando redução na qualidade do produto e comprometendo sua utilização. Nesse contexto, deve-se priorizar a escolha de espécies que 
apresentem elevada durabilidade natural (OLIVEIRA et al., 2005).

Essa propriedade pode ser observada por meio da realização de ensaios de preferência alimentar, porém, trabalhos com térmitas abordam parâmetros como a mortalidade de indivíduos, a perda de massa e desgaste das peças de madeira (PAES et al., 2007; FRANÇA et al., 2016; STALLBAUN et al., 2017), desconsiderando a influência do ataque dos cupins nas outras propriedades tecnológicas da madeira, importantes para definir o uso adequado desse material.

O objetivo deste trabalho foi analisar a influência que a deterioração provocada por térmitas do gênero Nasutitermes em propriedades mecânicas das madeiras de Cordia americana, Mezilaurus itauba, Melia azedarach e Parapiptadenia rigida.

\section{MATERIAL E MÉTODOS}

\subsection{Matéria prima utilizada no estudo}

Para a realização do presente estudo, selecionaram-se indivíduos arbóreos das espécies Cordia americana (L) Gottschling J. S. Mill., Melia azedarach L. e Parapiptadenia rigida (Benth.) Brenan, em um fragmento de Floresta Estacional Decidual, localizado no município de Jaguari - RS. As amostras de Mezilaurus itauba Taub. ex Mez. foram adquiridas no comércio local.

A partir de tabuas das espécies florestais supracitadas, confeccionaram-se 80 corpos de prova ( 20 por espécie), com dimensões de 15 × 15 × $260 \mathrm{~mm}$, considerando a maior dimensão no sentido axial, ou paralelo às fibras. Das 20 amostras por espécie, 10 foram utilizadas no ensaio de deterioração biológica com térmitas e as outras 10 como grupo controle.

Após confeccionados, postaram-se os corpos de prova no interior de uma estufa laboratorial sem circulação forçada de ar à $60^{\circ} \mathrm{C}$ de temperatura, permanecendo até massa constante. Posteriormente, em uma balança analítica de precisão $(0,01 \mathrm{~g})$, efetuou-se a pesagem das amostras para obtenção dos seus pesos iniciais.

\subsection{Ensaio de deterioração biológica}

Conduziu-se o ensaio de biodeterioração das madeiras submetidas ao ataque de térmitas Nasutitermes conforme adaptação da norma ASTM D 3345 (2008). Para tanto, utilizaram-se 10 amostras de cada espécie (totalizando 40), sistematicamente distribuídas em 4 linhas, com espaçamentos de 2 e $5 \mathrm{~cm}$ nas linhas e entrelinhas, respectivamente.

As 40 amostras ficaram expostas ao ataque de térmitas por um período de 40 dias. Transcorrido o período de exposição, utilizando-se de um pincel e uma espátula, removeram-se cautelosamente os substratos (areia) e os térmitas remanescentes nos corpos de prova, encaminhando os mesmos novamente até a estufa, regulada à $60^{\circ} \mathrm{C}$, até massa constante, para a obtenção da massa final, e posterior cálculo da perda de massa.

Os corpos de prova deteriorados, juntamente com as amostras controle, foram acondicionados em uma câmara climatizada $\left(20^{\circ} \mathrm{C}\right.$ e $65 \%$ de umidade), almejando a obtenção do equilíbrio higroscópico (teor de umidade de equilíbrio de 12\%), para determinação dos parâmetros tecnológicos de interesse.
2.3 Propriedades tecnológicas das madeiras in natura e deterioradas

Inicialmente, determinaram-se as massas específicas básicas das madeiras (Equação 1) e as respectivas perdas de massa (Equação 2).

$$
\begin{aligned}
& \rho \mathrm{b}=\mathrm{M}_{0} / \mathrm{V}_{\mathrm{S}} \\
& \mathrm{PM}=\left[\left(\mathrm{M}_{\mathrm{I}}-\mathrm{M}_{\mathrm{F}}\right) / \mathrm{M}_{\mathrm{I}}\right] \times 10
\end{aligned}
$$

em que: $\rho b$ - massa específica básica $\left(\mathrm{g} / \mathrm{cm}^{3}\right) ; \mathrm{M}_{0}$ - massa da madeira seca em estufa à $103 \pm 2^{\circ} \mathrm{C}(\mathrm{g}) ; \mathrm{V}_{\mathrm{S}}$ - volume da madeira na condição saturada $\left(\mathrm{cm}^{3}\right)$; PM - perda de massa das amostras de madeiras deterioradas por térmitas Nasutitermes $(\%) ; \mathrm{M}_{\mathrm{I}}$ - massa das amostras de madeira antes do ataque de térmitas Nasutitermes ( $\mathrm{g}$ ); $\mathrm{M}_{\mathrm{F}}-$ massa das amostras de madeira após o ataque de térmitas Nasutitermes (g).

Foram realizados os ensaios de dureza Janka e flexão estática, o qual possibilitou à obtenção do módulo de elasticidade (MOE) e módulo de ruptura (MOR). Os ensaios foram conduzidos de acordo com a norma ASTM D 143 (2014), em uma máquina universal de ensaios EMIC, com células de carga de $300 \mathrm{kN}$.

No ensaio de dureza Janka obtidos os dados de tensão máxima na parte enterrada e na parte sem contato com o substrato. Os parâmetros relacionados às propriedades mecânicas foram obtidos de forma direta por meio do software do equipamento.

\subsection{Análise estatística dos resultados}

Os resultados foram avaliados por meio da análise da variância, a 5\% de probabilidade de erro, e posteriormente o teste de comparação de médias (Tukey). Visando a homogeneização dos dados, converteram-se os percentuais de perda de massa em arcsen (Equação 3), conforme sugerido por Steell e Torrie (1980), sendo PM - perda de massa (\%).

$$
\operatorname{Arcsen}(\sqrt{ } \mathrm{PM} / 100)
$$

(Equação 3)

\section{RESULTADOS}

As massas específicas básicas ( $\rho b)$ das espécies florestais estudadas são apresentadas na Tabela 1. Parapiptadenia rigida apresentou significativamente a maior densidade, seguida da Cordia americana, Mezilaurus itauba e Melia azedarach, respectivamente.

As espécies $M$. azedarach e $C$. americana apresentaram perda de massa inferior e significativa em relação à $P$. rigida e $M$. itauba.

Tabela 1. Valores médios de massa específica básica $(\rho b)$ e perda de massa (PM) das espécies estudadas.

Table 1. Average values of basic specific mass $(\rho b)$ and mass loss (PM) of studied species.

\begin{tabular}{lcc}
\multicolumn{1}{c}{ Espécie } & $\rho \mathrm{b}\left(\mathrm{g} / \mathrm{cm}^{3}\right)$ & $\mathrm{PM}(\%)$ \\
\hline M. itauba & $0,723 \mathrm{c}$ & $2,47 \mathrm{~b}$ \\
C. americana & $0,837 \mathrm{~b}$ & $1,17 \mathrm{a}$ \\
P. rigida & $0,908 \mathrm{a}$ & $2,15 \mathrm{~b}$ \\
M. azedarach & $0,597 \mathrm{~d}$ & $0,99 \mathrm{a}$ \\
\hline
\end{tabular}

em que: médias minúsculas nas colunas não diferem entre si, conforme teste Tukey, em 5\% de probabilidade de erro. 
A C. americana apresentou o maior módulo de ruptura, entretanto, não diferiu significativamente da $P$. rigida (Tabela 2). Quanto à deterioração provocada por térmitas no MOR, observam-se alterações não significativas em todas as espécies. As espécies $M$. itauba, C. americana e $P$. rigida apresentaram redução nessa propriedade $(1,70 \%, 2,35 \%$ e $0,09 \%$, respectivamente), enquanto a $M$. azedarach teve comportamento oposto (aumento de 6,20\%).

Em relação ao módulo de elasticidade (MOE), nota-se a ausência de diferenças significativas para as madeiras do grupo controle, observando-se o oposto para as madeiras das espécies deterioradas. A espécie $M$. itauba foi a única a não apresentar diferença significativa entre as madeiras dos grupos controle e deterioradas. Em todas as espécies observaram-se reduções no MOE: 17,14\% (M. itauba), 18,42\% (C. americana), 28,03\% (P. rigida) e 23,94\% (M. azedarach).

Já na Tabela 3 observa-se a existência de diferenças significativas na dureza Janka das madeiras dos grupos controle e deterioradas, tanto na posição exposta ao ar (topo), quanto na enterrada no substrato (base). Visualizaram-se os maiores valores de dureza nas espécies com maiores densidades ( $C$. americana e $P$. rigida), as quais não diferiram significativamente entre elas.

Tabela 2. Valores médios dos parâmetros relacionados ao ensaio de flexão estática para as madeiras do grupo controle e deterioradas por térmitas Nasutitermes.

Table 2. Average values of the parameters related to the static bending test for Woods of control group and deteriorated by Nasutitermes termites.

\begin{tabular}{lcc}
\hline \multicolumn{3}{c}{ MOR $(\mathrm{MPa})$} \\
\hline \multicolumn{1}{c}{ Espécie } & Controle & Deteriorada \\
\hline M. itauba & $115,83 \mathrm{~B} \mathrm{a}$ & $113,86 \mathrm{~B} \mathrm{a}$ \\
C. americana & $159,38 \mathrm{~A} \mathrm{a}$ & $155,63 \mathrm{~A} \mathrm{a}$ \\
P. rigida & $154,92 \mathrm{~A} \mathrm{a}$ & $154,78 \mathrm{~A} \mathrm{a}$ \\
M. azedarach & $99,64 \mathrm{~B} \mathrm{a}$ & $105,82 \mathrm{~B} \mathrm{a}$ \\
\hline \multicolumn{3}{c}{ MOE $(\mathrm{MPa})$} \\
\hline Espécie & Controle & Deteriorada \\
\hline M. itauba & $10.579,7 \mathrm{~A} \mathrm{a}$ & $8.766,5 \mathrm{AB} \mathrm{a}$ \\
P. rigida & $12.612,4 \mathrm{~A} \mathrm{a}$ & $10.288,6 \mathrm{~A} \mathrm{~b}$ \\
M. azedarach & $13.097,2 \mathrm{~A} \mathrm{a}$ & $9.426,2 \mathrm{AB} \mathrm{b}$ \\
\hline
\end{tabular}

Em que: médias maiúsculas nas colunas e minúsculas nas linhas não diferem entre si, conforme teste Tukey, em 5\% de probabilidade de erro.

Tabela 3. Valores médios de dureza Janka para as madeiras do grupo controle e deterioradas por térmitas Nasutitermes.

Table 3. Average values of Janka hardness for Woods of control group and deteriorated by Nasutitermes termites.

\begin{tabular}{lcc}
\hline \multicolumn{3}{c}{ Tensão máxima (MPa) - Topo } \\
\hline \multicolumn{1}{c}{ Espécie } & Controle & Deteriorada \\
\hline M. itauba & $30,64 \mathrm{~B} \mathrm{a}$ & $28,09 \mathrm{~B} \mathrm{a}$ \\
C. americana & $78,51 \mathrm{~A} \mathrm{a}$ & $74,76 \mathrm{~A} \mathrm{a}$ \\
P. rigida & $77,44 \mathrm{~A} \mathrm{a}$ & $71,67 \mathrm{~A} \mathrm{a}$ \\
M. azedarach & $40,76 \mathrm{~B} \mathrm{a}$ & $39,56 \mathrm{~B} \mathrm{a}$ \\
\hline \multicolumn{3}{c}{ Tensão máxima (MPa) - Base } \\
\hline \multicolumn{1}{c}{ Espécie } & Controle & Deteriorada \\
\hline M. itauba & $29,93 \mathrm{C} \mathrm{a}$ & $27,14 \mathrm{~B} \mathrm{a}$ \\
C. americana & $72,60 \mathrm{~A} \mathrm{a}$ & $69,17 \mathrm{~A} \mathrm{a}$ \\
P. rigida & $75,52 \mathrm{~A} \mathrm{a}$ & $69,66 \mathrm{~A} \mathrm{a}$ \\
M. azedarach & $44,67 \mathrm{~B} \mathrm{a}$ & $38,85 \mathrm{~B} \mathrm{a}$ \\
\hline
\end{tabular}

em que: médias maiúsculas nas colunas e minúsculas nas linhas não diferem entre si, conforme teste Tukey, em 5\% de probabilidade de erro.
$\mathrm{O}$ ataque de térmitas Nasutitermes acarretou redução na dureza, nas posições base e topo, das amostras deterioradas em relação aos respectivos grupos controle. No topo observou-se reduções de $8,32 \%, 4,78 \%, 7,45 \%$ e 2,94\% para as espécies $M$. itauba, C. americana, $P$. rigida e $M$. azedarach, respectivamente, enquanto na base, essas reduções foram de: 9,32\% (M. itauba), 4,72\% (C. americana), 7,76\% (P. rigida) e $13,03 \%$ (M. azedarach).

\section{DISCUSSÃO}

Carvalho (2002) relatou que para a espécie $P$. rigida a massa específica aparente (condição de $15 \%$ de umidade) encontra-se compreendida entre 0,75 à $1,00 \mathrm{~g} / \mathrm{cm}^{3}$. Baseandose na classificação descrita por Carvalho (1996), essa espécie e a Cordia americana enquadram-se na classe de madeiras pesadas $\left(0,800-0,950 \mathrm{~g} / \mathrm{cm}^{3}\right)$, enquanto Mezilaurus itauba e Melia azedarach inserem-se nas classes de madeiras moderadamente pesadas $\left(0,650-0,79 \mathrm{~g} / \mathrm{cm}^{3}\right)$ e leves $(0,50-$ $\left.0,64 \mathrm{~g} / \mathrm{cm}^{3}\right)$, respectivamente.

Devido a relação dessa propriedade física $\left(\rho_{\mathrm{b}}\right)$ com as propriedades mecânicas da madeira (TREVISAN et al., 2008), a mesma torna-se um parâmetro de grande relevância a ser considerado na qualificação da madeira (PALHARINI et al., 2014), principalmente no que tange para fins estruturais.

Considerando a PM, Stallbaun et al. (2017) determinaram um percentual médio de $2,19 \%$ para a madeira de Sclerolobium paniculatum deteriorada por térmitas Nasutitermes, demonstrando que a baixa perda de massa pode ser decorrente de outros fatores inerentes á espécie madeireira.

Semelhante ao ocorrido no presente estudo (principalmente quanto as espécies $M$. azedarach e $P$. rigida), Stallbaun et al. (2017) observaram que algumas árvores que apresentaram $\rho_{b}$ inferior, demonstraram maior resistência à deterioração por térmitas, atribuindo isso a parâmetros relacionados à idade, a qualidade do sítio florestal e aos extrativos presentes na madeira. Constata-se, portanto, que a durabilidade natural da madeira ao xilófago em questão não pode ser atribuída somente à sua densidade, fato destacado por Paes et al. (2007).

Nesse contexto, sabe-se que a resistência que a madeira oferece à deterioração por térmitas varia entre as diferentes espécies. Isso possivelmente está associado à propriedades físicas, como a densidade e a porosidade, anatômicas e químicas.

Quanto às variações recorrentes em parâmetros relacionados às propriedades mecânicas, as madeiras de $M$. itauba e $M$ azedarach possuem menor densidade quando comparadas com $C$. americana e $P$. rigida, e além disso, a madeira de $M$. itauba apresentou maior porcentagem de perda de massa em relação às demais.

Considerando a heterogeneidade e as variações recorrentes na madeira, atreladas principalmente à sua complexidade estrutural e de desenvolvimento, variações recorrentes em uma mesma propriedade tecnológica podem ocorrer entre diferentes espécies, bem como em uma mesma espécie (EVANGELISTA et al., 2010).

Fatores anatômicos tais como lenhos inicial e tardio, e a madeira adulta e juvenil, possuem influência decisiva nos parâmetros relacionados as propriedades mecânicas das madeiras, além da idade dos indivíduos. Isso possibilita, em partes, justificar os aumentos e as reduções recorrentes nos parâmetros relacionados ás propriedades mecânicas, em conjunto com a deterioração causada por térmitas dos 
componentes químicos principais, principalmente as hemiceluloses e a celulose.

Uma vez que térmitas possuem capacidade de degradar a celulose (SILVEIRA et al., 2015) e hemicelulose, escarificando a lignina, as modificações em suas quantidades e estruturas influenciam diretamente nos parâmetros tecnológicos relacionados às propriedades mecânicas das madeiras, corroborado com o argumentado por Braz et al. (2014).

Outro fator inerente ao ataque de térmitas é a abertura de galerias no interior da madeira (CORASSA et al., 2014) devido a remoção de componentes químicos, ocasionado assim decréscimo na resistência mecânica, aumentando a porosidade desse material pela redução da quantidade de material lenhoso.

\section{CONCLUSÕES}

A espécie mais resistente ao ataque de térmitas Nasutitermes no presente estudo foi a Melia azedarach, a qual não diferiu significativamente da Cordia americana, mesmo possuindo a menor massa específica básica frente às demais, indicando assim que essa resistência pode estar associada à sua composição química.

A deterioração acarretada por térmitas causou reduções nas propriedades mecânicas das madeiras deterioradas em comparação ao grupo controle, contudo, foi significativa apenas para o módulo de elasticidade das espécies Cordia americana, Parapiptadenia rigida e Mezilaurus itauba.

\section{REFERÊNCIAS}

AMERICAN SOCIETY FOR TESTING AND MATERIALS (ASTM). Standard test methods for small clear specimens of timber. ASTM D 143 - 94. Philadelphia, 2014.

AMERICAN SOCIETY FOR TESTING AND MATERIALS (ASTM). Standard Test method for laboratory evaluation of Wood and other cellulosic materials for resistance to termites. ASTM D 3345 - 74. West Conshohocke, 2008.

ARAUJO, H. J. B.; MAGALHÃES, W. L. E.; OLIVEIRA, L. C. Durabilidade de madeira de eucalipto citriodora (Corymbia citriodora (Hook.) K.D. Hill \& L.A.S. Johnson) tratada com CCA em ambiente amazônico. Acta Amazonica, v. 42, n. 1, p. 49-58, 2012. $<\mathrm{http} / / / \mathrm{dx}$.doi.org/10.1590/S0044-59672012000100006>.

BRAZ, R. L.; OLIVEIRA, J. T. S.; ROSADO, A. M.; VIDAURRE, G. B.; PAES, J. B.; TOMAZELLO FILHO, M.; LOIOLA, P. L. Caracterização anatômica, física e química da madeira de clones de Eucalyptus cultivados em áreas sujeitas à ação de ventos. Ciência da Madeira, Pelotas, v. 5, n. 2, p. 127-137, 2014. <DOI: 10.12953/2177-6830.v05n02a07>.

CARVALHO, P. E. R. Espécies Arbóreas Brasileiras. Colombo, PR: Embrapa Florestas, v. 1, 2003, 1039 p.

CARVALHO, A. Madeiras portuguesas, estrutura anatômica, propriedades utilizações. Instituto Florestal, v. 1, 1996.

CARVAlHO, P. E. R. Angico-Gurucaia. Circular técnica 58. Empresa Brasileira de Pesquisa Agropecuária Embrapa, Colombo, Paraná, 2002. 14 p.

CORASSA, J. N.; PIRES, E. M.; ANDRADE NETO, V. R.; TARIGA, T. C. Térmitas associados à degradação de cinco espécies florestais em campo de apodrecimento. Floresta e Ambiente, Seropédica, v. 21, n. 1, p. 78-84, 2014. $<\mathrm{http}$ //dx.doi.org/10.4322/floram.2014.014>.
EVANGELISTA, W. V.; SILVA, J. C.; DELlA LUCIA, R. M.; LOBO, L. M.; SOUZA, M. O. A. Propriedades físicomecânicas da madeira de Eucalyptus urophylla S. T. Blake no sentido radial e longitudinal. Ciência da Madeira, Pelotas, v. 1, n. 2, p. 01-19, 2010.

FRANÇA, T. S. F. A.; FRANÇA, F. J. N.; ARANGO, R. A.; WOODWARD, B. M.; ARANTES, M. D. C. Natural resistance of plantation grown African mahogany (Khaya ivorensis and Khaya senegalensis) from Brazil to wood-rot fungi and subterranean termites. International Biodeterioration \& Biodegradation, Elsevier, v. 107, p. 88-91, 2016. $<$ https://doi.org/10.1016/j.ibiod.2015.11.009>

MOTTA, J. P.; OLIVEIRA, J. T. S.; PAES, J. B.; ALVES, R. C.; DAMBROZ, G. B. V. Resistência natural da madeira de Tectona grandis em ensaio de laboratório. Ciência Rural, Santa Maria, v.43, n.8, p.1393-1398, 2013. $<$ http://dx.doi.org/10.1590/S0103-84782013005000097>.

OLIVEIRA, J. T. S.; SOUZA, L. C.; DELLA LUCIA, R. M.; SOUZA JUNIOR, W. P. Influência dos extrativos na resistência ao apodrecimento de seis espécies de madeira. Revista Árvore, Viçosa-MG, v. 29, n. 5, p. 819-826, 2005. $<$ http://dx.doi.org/10.1590/S0100-67622005000500017>.

PAES, J. B.; MELO, R. R.; LIMA, C. R. Resistência natural de sete madeiras a fungos e cupins xilófagos em condições de laboratório. Cerne, Lavras, v. 13, n. 2, p. 160-169, 2007.

PALHARINI, K. M. Z.; PAIVA, C. S.; CARVALHO, D. M.; GUIMARÃES JUNIOR, J. B.; PROTÁSIO, T. P. Avaliação das propriedades físicas em madeiras de Croton celtidifolius e Myracrodruon urundeuva. Enciclopédia Biosfera, Goiânia, v. 10, n. 19, p. 2982-2989, 2014.

RODRIGUES, W. F.; GOMES, G. C.; MEDEIROS, A. R. M.; BARBIERI, R. L. Espécies arbóreas da Serra dos Tapes: um resgate etnobotânico. Documentos, 190. Empresa Brasileira de Pesquisa Agropecuária - Embrapa, Embrapa Clima Temperado, Pelotas, 2007. 68 p.

SILVEIRA, A. G.; SANTINI, E. J.; TREVISAN, R.; CANCIAN, L. C.; MARIANO, L. G. Ocorrência e danos de térmitas na madeira de Acacia mearnsii (Fabaceae) em dois campos de apodrecimento (nota científica). Revista do Instituto Florestal, v. 27, n. 2, p. 183-189, 2015.

STALLBAUN, P. H.; BARAUNA, E. E. P.; PAES, J. B.; RIBEIRO, N. C.; MONTEIRO, T. C.; ARANTES, M. D. C. Resistência natural da madeira de Sclerolobium paniculatum Vogel a cupins em condições de laboratório. Floresta e Ambiente, Seropédica, v. 24, 6 p., 2017. $<$ http://dx.doi.org/10.1590/2179-8087.001316>.

STEEL, R. G. D.; TORRIE, J. H. Principles and procedures of statistic: a biometrical approach. 2. ed. New York: McGraw Hill, 1980. 633 p.

TREVISAN, R.; HASELEIN, C.R.; MELO, R.R.; STANGERLIN, D.M.; BELTRAME, R.; GATTO, D.A.; CALEGARI, L. Variação radial da massa específica básica da madeira de Eucalyptus grandis W. Hill ex Maiden. Floresta, Curitiba, n.3, p.553-559, 2008.

VENSON, I., SILVA GUZMÁN, J. A., FUENTES TALAVERA, F. J. \& RICHTER, H. G. 2008. Biological, physical and mechanical wood properties of paraíso (Melia azedarach) from a roadside planting at Huaxtla, Jalisco, Mexico. Journal of Tropical Forest Science, v. 20, n. 1, p 38-47, 2008. 